\title{
Informations sur les contributeurs et contributrices
}

\author{
The Dynamics of Wordplay $6 \& 7$
}

\section{Karine Abiven (Sorbonne Université)}

Maitre de conférence à Sorbonne Université et membre de l'Institut Universitaire de France, Karine Abiven est spécialiste de la langue et de la littérature françaises des XVII ${ }^{\mathrm{e}}$-XVIII ${ }^{\mathrm{e}}$ siècles. Elle a écrit sur les formes brèves de l'écriture non fictionnelle (L'Anecdote ou la fabrique du petit fait vrai, Paris, Classiques Garnier, 2015), a dirigé plusieurs numéros de revue sur l'écriture de l'actualité et de l'histoire (Littératures classiques 78 et 94). Elle se consacre désormais à l'exploration numérique des libelles sous la Fronde (les « mazarinades »).

\section{Salvatore Attardo (Texas A\&M University-Commerce)}

Salvatore Attardo (Doctorat de l'Université de Purdue, 1991) est professeur de Linguistique à l'Université de Texas A\&M-Commerce. Il a publié deux livres : Linguistic Theories of Humor, en 1994, et Humorous Texts, en 2001. Il a été directeur de HUMOR : International Journal of Humor Research pendant 10 ans. Il a également été directeur de l'Encyclopedia of Humor Studies (Sage, 2014) et de l'Handbook of Language and Humor (Routledge, 2017). Il a publié plus de 100 articles sur différents sujets de sémantique et de pragmatique.

\section{Pauline Beaucé (Université Bordeaux Montaigne)}

Pauline Beaucé (née en 1986) est Docteur en Langue et Littérature françaises (2011) et a été postdoctorante dans le projet ERC DramaNet (FU Berlin, 2013). Depuis 2015, elle est Maître de conférences en études théâtrales à l'Université Bordeaux Montaigne. Ses recherches portent sur l'histoire des spectacles aux $\mathrm{XVIII}^{\mathrm{e}}$ et $\mathrm{XIX}^{\mathrm{e}}$ siècles, notamment l'histoire des formes spectaculaires (parodie, théâtre musical, pantomimes) et des lieux de divertissement (théâtres forains, cirque, wauxhall). Elle est l'auteur de Parodies d'opéra au siècle des Lumières, PUR, 2013, d'une trentaine d'articles et entrées de dictionnaires ainsi que d'éditions scientifiques de textes dramatiques inédits. Pauline Beaucé est membre du Réseau Dynamik des Wortspiels et participe au projet ANR Ciresfi sur la Comédie Italienne et les théâtres forains au XVIII ${ }^{\mathrm{e}}$ siècle (Université de Nantes, Françoise Rubellin). 


\section{Angelika Braun (Universität Trier)}

Angelika Braun a étudié la linguistique allemande et la phonétique à l'Université de Marbourg. Elle a obtenu son doctorat en 1988. De 1986 à 2000, elle a travaillé comme phonéticienne forensique au Bureau Criminal Fédéral et au Laboratoire Forensique de la Rhénanie-du-Nord-Westphalie. En 2000, elle a obtenu son Habilitation à l'Université de Marbourg à laquelle elle a travaillé de 2000 à 2009. La même année, elle a rejoint cette université. Depuis 2009, elle occupe le poste de Professeure de Phonétique générale et appliquée à l'Université de Trèves. Ses thèmes de recherche principaux sont les caractéristiques du locuteur et leur variabilité, ainsi que la sociophonétique, en particulier le langage et l'émotion, non-actual speech (ironie verbale) et les aspects phonétiques $\mathrm{du}$ jeu du mots. Pendant toute sa carrière académique, elle a maintenu un intérêt pour l'histoire des sciences phonétiques, comme le montrent ses publications dans ce domaine.

\section{François Chaix (acteur / metteur en scène, Paris)}

Après les cours d'Anicette Fray puis de Jean-Laurent Cochet, François Chaix entre à La Classe Supérieure d'Art Dramatique de la Ville de Paris (ESAD), qu'il quitte en 1989. Professionnel dès l'année suivante, il joue dans de nombreuses productions (Molière, Musset, Rostand, Brecht, Feydeau, etc.). Il est de nombreuses années comédien permanent de l'Auguste Théâtre-Cie Gilles Robin. Il est aussi de plusieurs spectacles de Jean-Marie Villégier (Le Fidelle de Pierre de Larivey, La Répétition interrompue de Charles Favart, etc.). Il rejoint Aurélie Rusterholtz en 2007 pour les lectures-spectacles du Grand T à Nantes, rendezvous annuel des perles rares de ce théâtre des XVII ${ }^{\mathrm{e}}$ et XVIII ${ }^{\mathrm{e}}$ siècles (Fuzelier, Soulas d'Allainval, Gueullette, Regnard, Piron, Boisfranc, Coypel, Autreau). En 2011, il joue au Québec dans Arlequin Sauvage de Delisle de la Drevetière dans une mise en scène de Thierry Pillon. Il co-signe et joue Spectacle Eclair en 2015, met en scène et joue Satyrics d'après Jean-Claude Grumberg en 2016, co-signe la mise en scène de Travaux - Les entretiens d'embauche de Jacques Jouet en 2017 et de Regardez le soleil pour moi... - Paparazzi de Matéi Visniec. Il crée Emmanuel P. fusillé pour l'exemple de Bernard Briais mis en scène par Pascale Sueur en 2018. On le retrouve au cinéma avec Michel Béna, Rodolphe Marconi, à la télévision avec Christophe Barraud, Jean-Teddy Philippe, Myriam Touzé, Philippe Roussel, Kevin Connor, Roger Young, etc. Il développe ces dernières années une intense activité d'artiste-interprète de la voix enregistrée. 


\section{Dirk Delabastita (Université de Namur)}

Dirk Delabastita enseigne la littérature anglaise et la théorie littéraire à l'Université de Namur ; il est également chercheur associé à la KU Leuven. Plusieurs de ses publications portent sur les jeux de mots dans l'œuvre de Shakespeare et sur la question de leur traduction vers d'autres langues. Il est l'auteur de l'ouvrage There's a Double Tongue (1993) et a (co-)édité les volumes suivants : European Shakespeares (avec Lieven D'hulst, 1993), Traductio. Essays on Punning and Translation (1997), Fictionalizing Translation and Multilingualism (avec Rainier Grutman, 2005), Shakespeare and European Politics (avec Jozef de Vos et Paul Franssen, 2008), Multilingualism in the Drama of Shakespeare and His Contemporaries (avec Ton Hoenselaars, 2015) et "Romeo and Juliet» in European Culture (avec Juan F. Cerdá et Keith Gregor, 2017). Il dirige la revue Target. International journal of Translation Studies avec Sandra Halverson et est aussi co-directeur d'un dictionnaire en ligne de termes littéraires (en néerlandais), intitulé Algemeen Letterkundig Lexicon (http://www.dbnl.org/ tekst/dela012alge01_01/index.php).

\section{Alex Demeulenaere (Universität Trier)}

Alex Demeulenaere est Maître de conférences («Akademischer Oberrat») en langues et littératures romanes à l'Université de Trèves. Il a publié au sujet des littératures (post)coloniales et (post)nationales dans les cultures francophones (France, Belgique, Afrique, Canada), des études en traduction, des récits de voyage et de la théorie littéraire (Said, de Certeau). Dans sa thèse de Doctorat (Louvain 2007), il s'est basé sur l'analyse du discours pour étudier l'ethos narratif et la construction de crédibilité scientifique dans les récits de voyage coloniaux français. En tant que membre de l'IRTG «Diversity", sa recherche actuelle se base sur des développements dans le même domaine (par exemple la posture) pour une étude diachronique de la littérature nationale et postnationale au Québec. Ayant acquis une large expérience didactique aux Universités de Louvain et de Trèves, il a également organisé des séminaires multidisciplinaires en coopération avec l'Université de la Sarre et est en charge d'un séminaire en master portant sur la littérature et l'interculturalité à l'Université de Luxembourg.

\section{Hélène Favreau (Université Catholique de l'Ouest, Angers)}

Enseignant-chercheur en Sciences du Langage à la Faculté des Humanités (Université Catholique de l'Ouest, Angers), ses domaines de recherche vont de la sociolinguistique française (sa thèse de Doctorat avait pour objectif d'étudier 
les attitudes linguistiques des locuteurs lambdas quant à la question de la norme et de la variété) à la lexicologie, en particulier à la création lexicale, d'un point de vue morphologique et sémantique. Ces recherches ont donné lieu à divers articles : "Linguistic norms and standards : towards social exclusion» (in Norms in Educational Linguistics. Oxford : Peter Lang, 2010) ; "Speakers' Attitudes to Language : Generators or Mirrors of Sociolinguistic Changes? (The French Example)» (in Forum for Anthropology and Culture 21, Saint-Pétersbourg: Russian Academy of Sciences, 2014); “"On s'keep in Sosh” ou l'exemple du français libéré dans et par la publicité » (in Voix Plurielles 12.1, 2015).

\section{Natalia Filatkina (Universität Trier)}

Natalia Filatkina a étudié la linguistique et la communication interculturelle à l'Université linguistique d'Etat de Moscou et à l'Université Humboldt de Berlin. Elle a obtenu un doctorat en linguistique allemande à l'Université de Bamberg. Depuis 2003, elle enseigne la linguistique historique allemande à l'Université de Trèves, où elle a dirigé le groupe de recherche « Le langage formulaïque historique et les traditions de communication ». En 2017, elle a obtenu l'Habilitation à la même université et y occupe en ce moment la position de professeure adjointe («außerplanmäßige Professorin ») ainsi que celle de "Akademieprofessorin » pour le langage et la culture médiévaux à l'Université de Trèves et à l'Académie des Sciences et de Littérature à Mayence. Ses intérêts de recherche principaux incluent le changement linguistique, le langage figuratif et formulaïque, la standardisation et la normalisation, le dialogue historique, la linguistique textuelle et discursive ainsi que les humanités numériques. Elle codirige également la collection « Formelhafte Sprache/ Formulaic language ».

\section{Dirk Geeraerts (KU Leuven)}

Dirk Geeraerts est professeur de linguistique à l'Université de Leuven, où il a fondé le centre de recherches «Quantitative Lexicology and Variational Linguistics » (Lexicologie quantitative et linguistique variationnelle). Ses principaux domaines de recherche incluent les champs interreliés de la sémantique lexicale et de la lexicologie, avec un intérêt descriptif particulier pour la variation sociale, un engagement méthodologique marqué dans les analyses de corpus, à partir $\mathrm{du}$ fondement théorique de la linguistique cognitive (Cognitive Linguistics). Directeur fondateur de la revue Cognitive Linguistics, il a joué un rôle majeur dans l'expansion internationale de la linguistique cognitive. Ses publications incluent les monographies suivantes: Paradigm and Paradox 
(1985), The Structure of Lexical Variation (1994), Diachronic Prototype Semantics (1997), Words and Other Wonders (2006), The Oxford Handbook of Cognitive Linguistics (2007) et Theories of Lexical Semantics (2010).

\section{Raymond W. Gibbs, Jr. (University of California, Santa Cruz)}

Raymond W. Gibbs, Jr. est un chercheur en sciences cognitives et ancien professeur distingué de psychologie à l'University of California, Santa Cruz. Ses intérêts de recherche se concentrent sur la cognition incarnée, la pragmatique et le langage figuratif. Il est l'auteur de presque 300 articles et chapitres de livres et il a publié de nombreux livres, y compris The poetics of mind : Figurative thought, language and understanding (1994), Intentions in the experience of meaning (1999), Embodiment and cognitive science (2006), Metaphor wars: Conceptual metaphor in human life (2017) et (avec Herb Colston) Interpreting figurative meaning (2012), tous publiés chez Cambridge University Press. Il est aussi rédacteur du livre Cambridge handbook of metaphor and thought (2008) (CUP) et rédacteur du journal Metaphor and Symbol.

\section{Peter Handler (Wirtschaftsuniversität Wien - WU)}

Peter Handler est Maître de conférences à l'Institut de Langues romanes au sein du Département de Langues étrangères en Économie et Gestion de l’Université de Sciences économiques de Vienne (Wirtschaftsuniversität Wien - WU). L'objet de ses recherches porte sur la stylistique (néologie, phraséologie, théorie du texte), la formation des mots, la créativité langagière, les langues de spécialités, les techniques de présentation et les nouveaux médias (phénomènes linguistiques et sémiotiques). Il enseigne le français et l'allemand économique et commercial. Sa thèse interdisciplinaire Wortbildung und Literatur s'intéresse aux aspects poétologiques de la formation des mots. Parmi ses récentes publications, on trouve les contributions suivantes pour des ouvrages de référence : "Word-formation and literature» dans Word-Formation (éd. par P. O. Müller, I. Ohnheiser, S. Olsen \& F. Rainer, Handbooks of Linguistics and Communication Science 40) et les chapitres «Business presentations » ainsi que «Company websites » dans Handbook of Business Communication. Linguistic Approaches (éd. par G. Mautner \& F. Rainer, Handbooks of Applied Linguistics 13).

\section{Joshua T. Katz (Princeton University)}

Joshua T. Katz est Cotsen Professor in the Humanities (Professeur avec chaire en Sciences Humaines), Professeur en Lettres Classiques et membre (ainsi que périodiquement directeur) du programme de Linguistique à l'Université de 
Princeton, où il enseigne depuis 1998, année de l'obtention de son Doctorat en Linguistique à Harvard. Ses publications récentes abordent des questions linguistiques, littéraires et culturelles dans des œuvres variées, allant de la poésie grecque du VIII ${ }^{\mathrm{e}}$ ou VII ${ }^{\mathrm{e}}$ siècle av. J.-C. d'Homère et d'Hésiode à la fiction expérimentale des $\mathrm{XX}^{\mathrm{e}}$ et $\mathrm{XXI}^{\mathrm{e}}$ siècles de Russell Hoban et Paul Kingsnorth en anglais. Il s'intéresse tout particulièrement à l'histoire et à la pratique des jeux de mots.

\section{Catherine Kerbrat-Orecchioni (Université de Lyon 2)}

Catherine Kerbrat-Orecchioni est Pr. honoraire de l'Université Lumière Lyon 2. Elle a également enseigné à titre de Pr. invitée à l'Université Columbia de New York, l'Université de Genève, et l'Université de Californie à Santa Barbara. De 2000 à 2005, elle a occupé la chaire « Linguistique des interactions » à l'Institut Universitaire de France. Ses domaines de spécialité sont la pragmatique, l'analyse du discours et l'analyse des conversations. Elle a publié dans ces domaines de nombreux articles et ouvrages, dont L'énonciation, L'implicite, Les interactions verbales (3 tomes), Les actes de langage dans le discours, La conversation, Le discours en interaction et en 2017 Les débats de l'entre-deux-tours des élections présidentielles françaises : Constantes et évolutions d'un genre.

\section{Michelle Lecolle (Université de Lorraine)}

Michelle Lecolle est Maitre de conférences HDR en Sciences du langage à l'Université de Lorraine-Metz (Crem/Centre de Recherches sur les médiations). Ses recherches portent sur la sémantique nominale en discours, sur les noms collectifs et les noms propres de groupes, sur la néologie sémantique et sur le sentiment linguistique. Publications sélectionnées : « Dénomination de groupes sociaux : approche sémantique et discursive d'une catégorie de noms propres » (in Franck Neveu, Peter Blumenthal, Linda Hriba, Annette Gerstenberg, Judith Meinschaefer et Sophie Prévost, dirs., Quatrième Congrès mondial de linguistique française. Berlin : SHS Web of Conferences, 2014, 2265-2281) ; « Jeux de mots et motivation : une approche du sentiment linguistique » (in Esme Winter-Froemel et Angelika Zirker, dirs., Enjeux du jeu de mots. Perspectives linguistiques et littéraires. Berlin \& Boston : De Gruyter, 2015, 217-243) ; "Some specific insights into wordplay form : sublexical vs lexical level » (in Sebastian Knospe, Alexander Onysko et Maik Goth, dirs., Crossing Languages to Play with Words : Multidisciplinary Perspectives. Berlin \& Boston : De Gruyter, 2016, 63-70) ; Noms collectifs humains en français : enjeux sémantiques, lexicaux et discursifs (soumis). 


\section{Jiaying Li (Université Paris Nanterre)}

Jiaying Li est doctorante en études théâtrales à l'Université Paris Nanterre. Sa thèse a pour sujet le jeu verbal dans le théâtre français au $\mathrm{XX}^{\mathrm{e}}$ siècle (Jarry, Ionesco, Tardieu, Novarina). Elle a publié plusieurs articles en Chine et en Europe notamment sur la question du jeu de langage dans le théâtre « non dramatique ». Parmi ses dernières publications : «Le silence moderne dans «l'écriture à trou de Valère Novarina » (Quêtes littéraires 7, 2017), "Les enjeux du théâtre radiophonique de Jean Tardieu » (Revue Sciences / Lettres, 2017), « De l'idiolecte novarinien à l'individualisme linguistique » (in M. Viegnes et J. Rime, dirs., Représentations de l'individu en Chine et en Europe francophone, Alphil-Presses universitaires suisses, 2015). Elle est également traductrice en chinois du livre de Madeleine Bertaud: François Cheng : Un cheminement vers la vie ouverte (Hermann, 2011).

\section{Elena Meteva-Rousseva (Université de Sofia « St. KI. Ohridski »)}

Elena Meteva-Rousseva est Maître de conférences HDR au Département d'études romanes de l'Université de Sofia «St. Kliment Ohridski ». Domaines de recherche: analyse discursive de la presse, discours rapporté dans la presse, histoire et théorie de la traduction, traduction de l'humour. Publications : «Le comique - défi linguistique et extralinguistique pour le traducteur" (in A. Tchaouchev et al., éds., Traduction et communication interculturelle. Presses universitaires «St. Kl. Ohridski », 2010, 98-108) ; "Calembours, expressions figées détournées - comment y faire face en traduction » (RumeliDE Journal of language and Literature Studies 2016/6, April, Special Issue 2, 33-42) ; «L'humour dans Les Carnets du major Thompson de Pierre Daninos et les problèmes que celui-ci soulève en traduction anglaise et bulgare » (in Sophie Anquetil et al., éds., Autour des formes implicites. Presses Universitaires de Rennes, 2017, 293-304).

\section{Claudine Moulin (Universität Trier)}

Claudine Moulin est Professeure de Linguistique historique allemande à l'Université de Trèves et directrice scientifique du Trier Center for Digital Humanities. Elle a étudié la philologie anglaise et allemande à Bruxelles et à Bamberg. Après sa thèse de doctorat (1990) portant sur l'orthographe du haut allemand précoce, elle a été chercheuse à l'Université de Bamberg, où elle a obtenu son Habilitation à diriger des recherches en philologie et linguistique allemandes en 1999. En tant que Heisenberg Fellow de la fondation allemande pour la recherche (DFG), elle a passé plusieurs séjours de recherche à Oxford $(1995,1997)$, où elle 
a travaillé sur des paratextes et des marginalia vernaculaires. En 2002, elle a été Professeure de Linguistique à l'Université de Luxembourg. Elle a également été professeure invitée à l'Ecole Pratique des Hautes Etudes EPHE/Sorbonne à Paris et chercheuse à l'Institut d'Etudes Avancées à Paris. Ses recherches portent sur la linguistique historique et le changement linguistique, les langues et la littérature médiévale, la grammaticographie, la lexicographie, la graphématique et les humanités numériques. Elle est également un des éditeurs de la revue Sprachwissenschaft et de la collection « Germanistische Bibliothek ».

\section{Cécile Pajona (Université Nice Sophia Antipolis)}

Cécile Pajona est doctorante en littérature au sein du laboratoire Bases Corpus Langage (UMR 7320) à Nice. Sa thèse, conduite sous la direction de Geneviève Salvan, porte sur les procédés de fictionnalisation dans l'œuvre romanesque de Boris Vian. À travers une approche pragma-énonciative des textes de Vian, elle interroge les figures de style et leur apport dans la construction de l'univers fictionnel vianesque. Le corpus se prête particulièrement à l'étude des jeux de mots et de l'humour ainsi qu'à leur apport et leur rendement pragmatique. Ses articles essaient majoritairement d'appréhender la particularité du style vianesque.

\section{Astrid Poier-Bernhard (Karl-Franzens-Universität Graz)}

Depuis son habilitation en 2010, portant sur la littérature à contrainte contemporaine en France et en Italie, Astrid Poier-Bernhard est Professeure de Littératures romanes à l'Institut des Études Romanes de Graz. Dans sa thèse de Doctorat, un second livre et de nombreux articles, elle s'est consacrée à l'œuvre de Romain Gary (Romain Gary - Das brennende Ich. Literaturtheoretische Implikationen eines Pseudonymenspiels. Tübingen : Niemeyer, 1996 ; Romain Gary im Spiegel der Literaturkritik. Frankfurt am Main \& Wien : Lang, 1999). Depuis 1998 une grande partie de ses travaux de recherche se réfèrent à l'Oulipo et la littérature potentielle. Ce sujet constitue aussi le thème principal d'un essai littéraire dans le genre du pastiche, Viel Spaß mit Haas! (Wien : Sonderzahl, 2003) et du livre (académique) Texte nach Bauplan. Studien zur zeitgenössischen ludischmethodischen Literatur in Frankreich und Italien (Heidelberg: Winter, 2012). Astrid Poier-Bernhard s'engage dans de nombreuses coopérations avec des institutions culturelles et des artistes. En 2013, elle a été cooptée par le groupe Oplepo (Opificio di letteratura potenziale). 


\section{Alain Rabatel (Université de Lyon 1)}

Alain Rabatel est Professeur de Sciences du Langage à l'Université de Lyon 1, membre de l'UMR ICAR (Université de Lyon 2). Spécialiste d'énonciation, de linguistique textuelle et d'analyse des discours, il est l'auteur de 5 ouvrages, de plus de 160 articles et il a (co)dirigé une vingtaine d'ouvrages ou de numéros de revues. Alain Rabatel s'est d'abord fait connaître pour ses travaux sur les points de vue, l'empathie et la polyphonie dans les récits (Une histoire du point de vue, CELTED / Klincksieck, 1997 ; La construction textuelle du point de vue, Delachaux et Niestlé, 1998). Il s'est ensuite intéressé aux liens entre argumentation indirecte, effacement énonciatif et points de vue (Argumenter en racontant, Deboeck-Duculot, 2004, Homo Narrans. Pour une analyse énonciative et interactionnelle du récit (2 vol.), Lambert-Lucas, 2008). Il travaille aussi sur les figures à partir de la notion de points de vue en confrontation (Langue française 160, Le Français Moderne 79(1), Vox romanica 71 et 74, Tranel 61-62 et dans deux publications du réseau DWP 2 et 4). A. Rabatel a également publié de nombreux articles sur les discours religieux et médiatiques, autour des questions de responsabilité et de prise en charge énonciative, ainsi que sur des corpus d'interactions orales en contexte didactique, dégageant diverses postures de co-, sur- et sous-énonciation, à la charnière des problématiques cognitives, énonciatives et interactionnelles. Voir également: http://www.icar.cnrs.fr/ membres/arabatel.

\section{Anda Rădulescu (Universitatea din Craiova)}

Anda Rădulescu (Université de Craiova, Roumanie) est professeur à la Faculté des Lettres, Département de Français. Auteurs de 3 livres de traductologie et de 4 de syntaxe du français, elle a publié une centaine d'articles dans des revues nationales et internationales de spécialité et a participé à une trentaine de colloques internationaux. Ses préoccupations pour l'étude des formes de l'humour et pour les mécanismes des jeux de mots sont plus récentes (2013). En 2017, elle a co-organisé un colloque international à Craiova, ayant pour objet l'humour chez San-Antonio, à l'œuvre duquel elle porte un intérêt particulier. Elle est directrice de la revue Annales de l'Université de Craiova. Série Langues et Littératures romanes et fait partie du comité scientifique des revues Translationes (Université de l'Ouest de Timișoara), Colocvium (Craiova), Argotica (Université de Craiova) et Annals of the University of Craiova, Series: Philology, Applied Foreign Languages. 


\section{Lisa Roques (Université Bordeaux Montaigne)}

Lisa Roques consacre sa thèse de Doctorat (en Histoire, Langues et Littérature ancienne) aux fragments d'Ion de Chios, un auteur polymathe du V $V^{\text {e }}$ siècle. Ces recherches articulent aux enjeux littéraires et stylistiques des analyses sociales et politiques. La présentation de ces différents aspects a donné lieu à différentes publications (« De Cimon à Périclès : un regard insulaire », in Elite und Krise in antiken Gesellschaften/Élites et crises dans les sociétés antiques, Collegium Beatus Rhenanus, Vol. 5, 47-57. Stuttgart : Franz Steiner Verlag, 2016, ou « Ion à la table d'Athénée ", in S. Trousselard and S. Coin-Longeray (eds.), Les intentions de la citation. Les Cahiers d'ALLHiS 4, 2016, 37-58) et interventions dans des colloques ou journées d'étude («Quelques vers de Chios... », Internationale Konferenz: «Die literarische Form», Westfälische Wilhelms-Universität, Münster, octobre 2015, ou «L'homme qui ne savait pas jouer de la cithare: rumeur et arborescence d'Ion de Chios à Saint Augustin », Journée d'étude: «Trames arborescentes II », Tours, décembre 2016). Titulaire de l'Agrégation en Lettres Classiques, elle exerce aussi à plein temps dans un collège relevant de l'éducation prioritaire en région parisienne.

\section{Catherine Ruchon (Université Paul-Valéry-Montpellier III, Membre associé du laboratoire Pléiade)}

Docteure en sciences du langage spécialisée en analyse du discours, enseignante à Paul-Valéry Montpellier III, Catherine Ruchon travaille en particulier sur l'expression de la souffrance, dans différents domaines, et notamment celui de la maternité, de la naissance, et du deuil. C'était le sujet de sa thèse : «Des vertus antalgiques du discours? L'expression de la douleur et de l'attachement dans les discours sur la maternité » (2015, dirigée par Marie-Anne Paveau). Plus largement, ses thèmes de recherche sont actuellement l'analyse du discours animal, l'analyse des discours funéraires (autour de l'humain et de l'animal), les discours sur l'identité, la linguistique populaire, l'analyse de discours numérique, l'interactivité et l'éthique. Elle a écrit plusieurs articles sur ces sujets dont «Lexique, catégorisation et représentation: les reformulations métalinguistiques dans le discours animaliste " (Les carnets du Cediscor, numéro spécial, 2018, à paraître), « Une situation d'interlocution spécifique : les discours numériques du parent endeuillé à son enfant décédé » (Semen 45, 2018, à paraître), "L'être et le nom : éthique de la nomination dans le cadre du deuil périnatal », (Langage \& Société 163, 2018, 101-119) et "Identité numérique de parents endeuillés. Le pseudonyme comme pratique de deuil » (dans Thierry Guilbert et Pascaline Lefort, dirs., Discours et (re)constructions identitaires. Presses Universitaires du Septentrion, 2017, 133-148). 


\section{Aurélie Rusterholtz (actrice / metteuse en scène, Paris)}

Après l'École Nationale d'Art Dramatique du T.N.S., dont elle sort en 1993, elle joue notamment Ibsen (Hedda Gabler mis en scène par Gloria Paris), Maeterlinck (Pelléas et Mélisande mis en scène par Pierre Guillois), Marivaux (La Fausse Suivante mis en scène par Gloria Paris), Molière (Les Femmes savantes mis en scène par Isabelle Moreau et Gloria Paris), Destouches (Les Philosophes amoureux mis en scène par Jean-Marie Villégier), Emmanuel Bourdieu (Je crois? mis en scène par Denis Podalydès) mais aussi Sénèque, Brecht, etc. Maîtrisant la langue des signes, elle joue dans $K$. Lear, spectacle en langue française et en langue des signes mise en scène par Marie Montegani à l'International Visual Theater de Paris, dirigé par Emmanuelle Laborit, en 2007, avec tournée en Corée. Elle participe à la recréation mondiale d'Arlequin sauvage de Delisle de la Drevetière au Québec (2011) dans une mise en scène de Thierry Pillon. De 2004 à 2012, Aurélie Rusterholtz est invitée chaque année au Grand T de Nantes pour des lectures-spectacles de textes rares des XVII ${ }^{\mathrm{e}}$ et XVIII ${ }^{\mathrm{e}}$ siècles qu'elle assure seule et depuis 2007 avec François Chaix. Au cinéma, elle travaille avec Claudio Descalzi (Ghost in the machine), Luc Besson (Adèle Blansec), Emmanuel Bourdieu (Edouard Drumont). En 2014 elle fonde à Paris LESGENSDU4AVRIL, Compagnie-école de Théâtre, pour laquelle elle co-signe Spectacle Eclair en 2015, joue Satyrics d'après Jean-Claude Grumberg mis en scène par F. Chaix en 2016, co-signe la mise en scène de Travaux d'après Musée haut - musée bas de Jean Michel Ribes en 2017 et de Regardez le soleil pour moi... d'après La mastication des morts de Patrick Kermann, en 2018.

\section{Jean-François Sablayrolles (Université Paris 13)}

Ancien agrégé de grammaire, Jean-François Sablayrolles a consacré sa thèse à la néologie du français contemporain (Paris 8, 1996). Maître de conférences à Limoges (1997- 2002) puis à Paris 7 (2002-2005), il a soutenu l'HDR en 2004 et exercé les fonctions de PU à Paris 13 SPC de 2006 à 2016. Il a été membre, successivement, des laboratoires et groupes de recherche suivants : CERES de Limoges, CIEL de Paris 7, LDI UMR 7187 puis HTL UMR 7597. Ses recherches portent principalement sur la néologie du français contemporain, dont les emprunts et leurs équivalents autochtones. Il a fait paraître en 2000 La Néologie en français contemporain (Champion), Les néologismes (avec Jean Pruvost, que sais-je ? PUF, 2003, 3 éd. 2016), L'innovation lexicale (Champion, 2003, actes du colloque de Limoges 2001), La Fabrique des mots français (Lambert Lucas 2016, actes colloque de Cerisy 2015 avec Ch. Jacquet-Pfau), Les néologismes, créer des mots français aujourd'hui (Garnier/le Monde, 2017), et a fondé, en 2006, et dirige, avec John Humbley, la revue Neologica (Classiques Garnier). 


\section{Astrid Schmiedel (Sorbisches Institut - Serbski Institut Bautzen)}

Astrid Schmiedel a étudié la phonétique et la linguistique computationnelle à l'Université de Trèves, où elle a obtenu son Doctorat en 2016 avec une thèse sur la phonétique du discours ironique. De 2010 à 2014, elle a travaillé comme assistante de recherche au Département de phonétique de l'Université de Trèves. Après avoir occupé divers postes administratifs dans la fonction publique, elle a rejoint l'Institute Sorabe - Serbski institut à Bautzen en 2018. Dans le domaine du traitement des données scientifiques, elle travaille sur des sujets liés au traitement automatique du langage naturel, sur les processus de digitalisation et sur la linguistique de corpus, en particulier dans les deux langues minoritaires, le bas sorabe et le haut sorabe.

\section{Monika Schmitz-Emans (Ruhr-Universität Bochum)}

Monika Schmitz-Emans est Professeure de Littérature générale et comparée à l'Université de Bochum. Elle a fait des études de philologie allemande, de philosophie, de philologie italienne et de sciences de l'art à Bonn. Elle a soutenu une thèse de Doctorat sur Jean Paul et ses idées sur une théorie de la langue (Schnupftuchsknoten oder Sternbild. Jean Pauls Ansätze zu einer Theorie der Sprache) en 1986 ainsi qu'une thèse d'Habilitation à diriger des recherches (Schrift und Abwesenheit. Historische Paradigmen zu einer Poetik der Entzifferung und des Schreibens) en 1995. Elle a publié de nombreux articles sur la littérature $\mathrm{du} \mathrm{XVIII}^{\mathrm{e}}$ au XXI ${ }^{\mathrm{e}}$ siècle, sur les relations texte/image dans la littérature, sur des questions de la théorie de la littérature et sur les formes littéraires du livre. Ses publications les plus récentes comprennent une monographie sur les bandes dessinées de littérature (Literatur-Comics. Adaptationen und Transformationen der Weltliteratur, 2012) et l'édition d'un ouvrage collectif sur la littérature comparée au sein d'un espace linguistique (Komparatistik sprachhomogener Räume. Konzepte, Methoden, Fallstudien, 2017) en collaboration avec Natalia Bakshi et Dirk Kemper.

\section{Gesa Schole (Eberhard Karls Universität Tübingen et Universität Trier)}

Gesa Schole a obtenu un diplôme en études en traduction (portugais, espagnol, droit) à l'Université Ruprecht Karls de Heidelberg et un diplôme en sciences du langage à l'Université de Bremen. Pendant ses études à l'Université Nova de Lisbonne au Portugal, elle s'est spécialisée en littérature africaine et portugaise. Dans son travail de fin d'études, elle a analysé la traduction allemande du roman de Mia Couto intitulé $A$ varanda do frangipani. Aux Universités de Tübingen et Trèves, elle a enseigné la traduction, la sociolinguistique, l'ambi- 
guïté et la pragmatique. Elle termine en ce moment un Doctorat portant sur L'ambiguité à l'interface sémantico-pragmatique dans des dialogues espagnols et allemands au sein du groupe de formation en recherche [GRK 1808] «Ambiguïté : production et perception » à l'Université de Tübingen. Ses intérêts de recherche vont de la linguistique cognitive, la cognition spatiale et les prépositions à la signification pragmatique, l'étude des dialogues et l'ambiguïté.

\section{Françoise Sullet-Nylander (University of Stockholm)}

Françoise Sullet-Nylander est Professeure Titulaire au Département d'Etudes romanes et classiques de l'Université de Stockholm où elle enseigne la langue et l'analyse du discours françaises. Depuis son doctorat, ses travaux de recherche ont porté sur les problématiques du discours rapporté, de la reformulation et des jeux de langage dans les textes journalistiques. Elle a co-dirigé plusieurs ouvrages, Le Français parlé des médias (2007), La Linguistique dans tous les sens (2011), Discours rapporté, genre(s) et médias et Le discours rapporté : une question de genre? (2015) et Political Discourses at the Extremes : Expressions of Populism in Romance Speaking Countries (à paraître). Ses travaux actuels touchent aux stratégies de questionnement des journalistes, au discours rapporté, aux termes d'adresse et aux phénomènes de nomination dans les débats présidentiels français et sur l'hétérogénéité énonciative dans les textes journalistiques. Depuis 2014, elle conduit un projet interdisciplinaire sur les discours politiques dans les pays de langue romane (ROMPOL).

\section{Giovanni Tallarico (Università degli studi di Verona)}

Giovanni Tallarico est Maître de conférences en Langue et Traduction Françaises à l'Université de Vérone. Ses intérêts de recherche et ses publications portent sur la lexicologie (notamment les néologismes et les emprunts lexicaux), la lexicographie bilingue, le langage du sport et la traductologie. En 2013, il a coordonné (avec Michela Murano) un numéro des Études de linguistique appliquée (Les dictionnaires bilingues et l'interculturel 170, avril-juin 2013) et il est l'auteur du volume $L a$ dimension interculturelle du dictionnaire bilingue (Paris, Champion, 2016). Il participe au projet international Néoveille - plateforme de repérage, analyse et suivi des néologismes en sept langues, coordonné par Emmanuel Cartier (Université Paris 13 - Sorbonne Paris Cité).

\section{Verena Thaler (Universität Mannheim)}

Verena Thaler, Docteure en Linguistique romane, est enseignante-chercheuse à l'Université de Mannheim, Allemagne. Ses domaines de recherche comprennent 
la pragmatique, la politesse verbale, la linguistique interactionnelle, les particules pragmatiques et la linguistique des médias, en particulier l'analyse de la communication sur Internet. Elle a publié dans ces domaines de nombreux travaux de recherche, dont deux monographies (Chat-Kommunikation im Spannungsfeld zwischen Oralität und Literalität, 2003; Sprachliche Höflichkeit in computervermittelter Kommunikation, 2012) et différents articles dans des revues internationales. Elle a également co-édité un ouvrage collectif sur l'onomastique commerciale (Kontrastive Ergonymie. Romanistische Studien zu Produktund Warennamen, 2013) ainsi qu'un ouvrage sur les tendances actuelles en linguistique des médias (Medienlinguistik 3.0. Formen und Wirkung von Textsorten im Zeitalter des Social Web, 2016).

\section{Esme Winter-Froemel (Universität Trier)}

Esme Winter-Froemel est Professeure de Linguistique romane à l'Université de Trèves. Ses recherches portent sur le changement linguistique, la linguistique de contact, la sémantique lexicale et l'ambigüité (voir entre autres sa monographie sur les emprunts lexicaux, Entlehnung in der Kommunikation und im Sprachwandel. Theorie und Analysen zum Französischen, 2011, et les volumes codirigés Diskurstraditionelles und Einzelsprachliches im Sprachwandel/ Tradicionalidad discursiva e idiomaticidad en los procesos de cambio lingüístico, 2015, avec Araceli López Serena, Álvaro Octavio de Toledo y Huerta et Barbara FrankJob, et Cognitive Contact Linguistics, sous presse, avec Eline Zenner et Ad Backus). Dans ses travaux récents, elle s'intéresse en particulier aux changements sémantiques et pragmatiques qui accompagnent les emprunts linguistiques ainsi qu'au rôle de l'ambigüité et des traditions discursives dans les processus de changement linguistique. Un autre thème central de sa recherche est représenté par les jeux de mots. Depuis 2013, elle dirige le réseau scientifique « La dynamique du jeu de mots : contact linguistique, innovation linguistique, interaction locuteur-auditeur » financé par la Fondation allemande pour la recherché (DFG). De plus, elle est membre de l'école doctorale « Ambiguität Produktion und Rezeption » (Graduiertenkolleg 1808, Université de Tübingen).

\section{Ilias Yocaris (Université Côte d'Azur)}

Né en 1971, Ilias Yocaris est Maître de conférences en Littérature française à l'UCA (Université Côte d'Azur). Ses recherches portent essentiellement sur le roman français du XIX ${ }^{\mathrm{e}}-\mathrm{XXI}^{\mathrm{e}}$ siècle (Hugo, Zola, Roussel, Proust, Giono, RobbeGrillet, Simon, Littell), la sémiotique du discours littéraire et les fondements conceptuels des fictions postmodernes (littéraires et filmiques). Il a publié entre 
autres L'Impossible totalité : une étude de la complexité dans l'œuvre de Claude Simon (Toronto, Paratexte, 2002) et Style et semiosis littéraire (Paris, Classiques Garnier, «Investigations stylistiques », 2016).

\section{Eline Zenner (KU Leuven)}

Eline Zenner est Professeure agrégée (assistant professor) à l'Université de Leuven (KU Leuven, Campus Bruxelles), où elle enseigne la maîtrise du néerlandais à de futurs interprètes, traducteurs, à des experts de la communication multilingue et à des journalistes. Elle a étudié la linguistique des langues germaniques (néerlandais, anglais) à la KU Leuven (master en 2007). En mai 2013, elle soutient sa thèse intitulée « Cognitive Contact Linguistics. The macro, meso, and micro influence of English on Dutch » (La linguistique de contact cognitive. L'influence macro, meso et micro de l'anglais sur le néerlandais). Ses domaines de recherche incluent les apports mutuels entre la linguistique cognitive usage-based et la linguistique de contact, la variation entre usage standard et variétés linguistiques vernaculaires dans l'acquisition du langage, et la différentiation sémantique dans la variation lexicale, qu'elle étudie dans une perspective à la fois théorique et appliquée. 
